\title{
Optimum take-off angle in the standing long jump
}

\author{
Masaki Wakai ${ }^{a}$ and Nicholas P. Linthorne ${ }^{\text {b,* }}$ \\ ${ }^{a}$ School of Exercise and Sport Science, The University of Sydney, PO Box 170, \\ Lidcombe, NSW 1825, Australia \\ ${ }^{b}$ School of Sport and Education, Brunel University, Uxbridge, Middlesex, \\ UB8 3PH, United Kingdom
}

\begin{abstract}
The aim of this study was to identify and explain the optimum projection angle that maximises the distance achieved in a standing long jump. Five physically active males performed maximum-effort jumps over a wide range of take-off angles, and the jumps were recorded and analysed using a 2-D video analysis procedure. The total jump distance achieved was considered as the sum of three component distances (take-off, flight, and landing), and the dependence of each component distance on the take-off angle was systematically investigated. The flight distance was strongly affected by a decrease in the jumper's take-off speed with increasing take-off angle, and the take-off distance and landing distance steadily decreased with increasing take-off angle due to changes in the jumper's body configuration. The optimum take-off angle for the jumper was the angle at which the three component distances combined to produce the greatest jump distance. Although the calculated optimum take-off angles $\left(19-27^{\circ}\right)$ were lower than the jumpers' preferred take-off angles $\left(31-39^{\circ}\right)$, the loss in jump distance through using a sub-optimum take-off angle was relatively small.
\end{abstract}

PsycINFO classification: 4010

Keywords: Biomechanics; Projectile; Standing long jump; Take-off angle.

* Corresponding author. Tel +44 1895 266479: fax +44 1895269769

Email address: nick.linthorne@brunel.ac.uk (N.P. Linthorne) 


\section{Introduction}

The standing long jump is an athletic event that can trace its origins back as far as the Olympic Games of Ancient Greece. Although the standing long jump was discontinued from the Modern Olympic Games after 1912, it remains in common use as a test of explosive leg power. It is used as a physical aptitude test in vocations such as firefighting, law enforcement, and the military, and many sports coaches use it to monitor an athlete's response to a training program. The standing long jump has high correlations with isokinetic measures of leg strength, and it is a good predictor of sprint and long jump performance (Wiklander \& Lysholm, 1987).

One minor limitation in using the standing long jump as a physiological or performance test is that it is a moderately complex movement. To achieve the best possible performance a jumper must execute a coordinated pattern of countermovement, forward rotation of the whole body, and a double-arm swing. The magnitude and sequencing of the movements must ultimately project the jumper's centre of mass at high speed and at an appropriate angle to the horizontal. Studies of standing long jumps by adult males have yielded projection angles of 29$38^{\circ}$, but the biomechanical reasons for this choice of projection angle are not well understood (Aguado, Izquierdo, \& Montesinos, 1997; Ashby \& Heegaard, 2002; Horita, Kitamura, \& Kohno, 1991). The purpose of the present study was to identify the optimum projection angle in the standing long jump and to explain the biomechanical rationale for this optimum angle.

In the flight phase of the standing long jump the centre of mass of the jumper behaves like a projectile in free flight. However, the optimum projection angle that maximises the distance of the jump is not expected to be $45^{\circ}$. A projection angle of $45^{\circ}$ is only appropriate if the magnitude of the projection speed generated by the jumper is the same for all projection angles. In the long jump, javelin, and shot put, the projection speed of the athlete (or implement) is known to decrease with increasing projection angle (Linthorne, 2001; Linthorne, Guzman, \& Bridgett, 2005; Red \& Zogaib, 1977). That is, the athlete has a bias towards the production of horizontal speed, and this bias reduces the optimum projection angle to below $45^{\circ}$.

In the shot put, the decrease in projection speed arises partly because a higher projection angle requires a greater fraction of the athlete's muscular force to be expended in overcoming the weight of the shot, and partly because the structure of the human body favours the production of putting force in the horizontal direction more than in the vertical direction. In the long jump the athlete uses a fast run-up to produce a high horizontal speed, and then plants the take-off leg on the ground to convert some of this speed to vertical speed. However, high take-off angles require a progressively slower run-up speed to enable sufficient time for the jumper to generate the necessary upward impulse. The overall result is that the projection speed decreases rapidly with increasing projection angle.

In the shot put and long jump the athlete's optimum projection angle may be calculated by substituting a mathematical expression for the relation between the projection speed and the projection angle into the equation for the range of a projectile in free flight (Linthorne, 2001; Linthorne et al., 2005). This method produces optimum projection angles that are in good agreement with measured competition projection angles (about $36^{\circ}$ in the shot put, and about $21^{\circ}$ in the long jump). 
In the present study the optimum take-off angle in the standing long jump was calculated using a method similar to that used by Linthorne et al. (2005) for the long jump. Five physically active males performed maximum-effort jumps over a wide range of take-off angles, and the take-off speed, take-off height, landing height, take-off distance, and landing distance for each jump were obtained from a 2-D video analysis of the jumps. The optimum take-off angle for the jumper was calculated by combining the equation for the total jump distance with the mathematical expressions for the relations between the jump variables and the takeoff angle. The calculated optimum take-off angle was then compared to the jumper's preferred take-off angle. Simple models were developed to explain the observed relations, and the influence of each variable on the optimum take-off angle was investigated.

\section{Component Distances}

In a standing long jump the jumper aims to project his body for maximum horizontal distance beyond a take-off line. The jumper starts from a static standing position and then generates a large take-off speed by using a countermovement coupled with a double-arm swing and a double-leg takeoff. The take-off is characterised by a large forward lean of the body, and during the flight phase the jumper swings the legs forward underneath the body in preparation for landing. The jumper usually lands with a prominent forward lean of the trunk and with the feet extended well ahead of the hips. To be credited with a successful jump the jumper must retain balance after landing and not fall backwards into the pit.

A standing long jump performance is quantified by the total jump distance, $d_{\text {jump }}$, which is the distance from the take-off line to the nearest break in the landing area made by the heels at landing. The jump distance may be considered as the sum of the take-off distance, $d_{\text {take-off }}$, the flight distance, $d_{\text {flight }}$, and the landing distance,

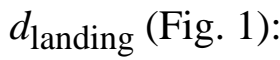

$$
d_{\text {jump }}=d_{\text {take-off }}+d_{\text {flight }}+d_{\text {landing }} \text {. }
$$

The take-off distance is the horizontal distance between the take-off line and the jumper's centre of mass at the instant of take-off, the flight distance is the horizontal distance the jumper's centre of mass travels from the instant of take-off to the instant of landing, and the landing distance is the horizontal distance between the jumper's centre of mass at the instant of landing and the break in the landing area nearest to the take-off line. All three component distances are expected to vary with changes in the jumper's take-off angle. Therefore, to calculate the optimum takeoff angle we need to have mathematical expressions for the dependence of each of the component distances on the take-off angle. 


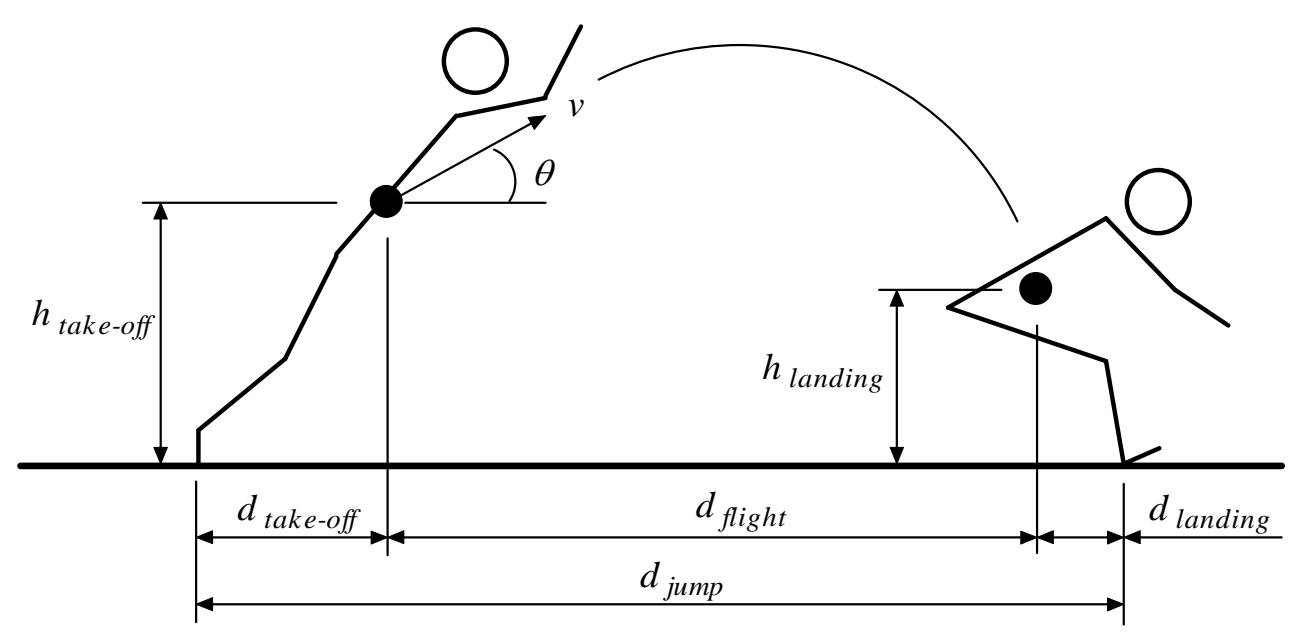

Fig. 1. Diagram of a standing long jump, showing contributions to the total jump distance.

\subsection{Flight distance}

In most jumps the flight distance makes the greatest contribution to the total jump distance. The flight distance may be calculated using the equation for the range of a projectile in free flight. During the flight phase of the jump the effects of gravity are much greater than those of aerodynamic forces and so the flight distance is given by (Hubbard, 2001)

$$
d_{\text {flight }}=\frac{v^{2} \sin 2 \theta}{2 g}\left[1+\left(1+\frac{2 g h}{v^{2} \sin ^{2} \theta}\right)^{1 / 2}\right],
$$

where $v$ is the take-off speed, $\theta$ is the take-off angle, and $g$ is the acceleration due to gravity. The relative take-off height, $h$, is given by

$$
h=h_{\text {take-off }}-h_{\text {landing }},
$$

where $h_{\text {take-off }}$ is the take-off height and $h_{\text {landing }}$ is the landing height (Fig. 1). When $h=0$, Eq. (2) reduces to the familiar expression for the range of a projectile launched from ground level over a horizontal plane, $d_{\text {flight }}=\left(v^{2} \sin 2 \theta\right) / g$. Therefore, in Eq. (2) the factor inside the curved brackets may be interpreted as a 'correction' to the flight distance due to the height difference between the take-off and landing.

It must be stressed that both the take-off speed and relative take-off height are not necessarily constant and may vary with changes in take-off angle. In the running long jump the jumper's take-off speed decreases rapidly with increasing take-off angle, and this reduces the optimum take-off angle to well below $45^{\circ}$ (Linthorne et al., 2005). If in the standing long jump the jumper's take-off speed also varies substantially with take-off angle, the relation between take-off speed and take-off angle, $v(\theta)$, can be expected to have a strong influence on the optimum take-off angle. In the running long jump the relative take-off height does not vary greatly with changes in take-off angle, and so $h(\theta)$ has only a minor effect influence on the optimum take-off angle. However, typical take-off speeds in the standing long jump (3.0-4.2 $\left.\mathrm{m} \cdot \mathrm{s}^{-1}\right)$ are much lower than in the long jump (8-10 $\left.\mathrm{m} \cdot \mathrm{s}^{-1}\right)$, and so the relative take-off height, even if it does not vary greatly with changes in takeoff angle, may play an important role in determining the optimum take-off angle in the standing long jump. 


\subsection{Take-off distance and landing distance}

The take-off distance and landing distance are also expected to vary with changes in take-off angle. If the take-off distance and landing distance decrease with increasing take-off angle the optimum take-off angle will be lower than if they remained constant. The greater the rate of decrease, the greater the reduction in the optimum take-off angle. In the running long jump the relations between the take-off distance and take-off angle, $d_{\text {take-off }}(\theta)$, and between the landing distance and takeoff angle, $d_{\text {landing }}(\theta)$, do not greatly influence the optimum take-off angle. However, in the standing long jump the take-off and landing distances make larger contributions to the total jump distance, and therefore the relations for $d_{\text {take-off }}(\theta)$ and $d_{\text {landing }}(\theta)$ may play an important role in determining the optimum take-off angle.

\section{Methods}

In this study the optimum take-off angle for the jumper was calculated by combining the equation for the flight distance of a body in free flight (Eq. 2) with mathematical expressions for the dependence of the jumper's take-off speed, takeoff height, and landing height on the take-off angle [i.e. $v(\theta), h_{\text {take-off }}(\theta)$, $\left.h_{\text {landing }}(\theta)\right]$. Mathematical expressions for the dependence of the take-off distance and landing distance [i.e. $d_{\text {take-off }}(\theta)$ and $d_{\text {landing }}(\theta)$ ] were also obtained, and then combined with the flight distance to calculate the total jump distance (Eq. 1). This method required intervention by the investigators to obtain measurements of the take-off and landing variables over a wide range of take-off angles, rather than just at the jumper's preferred take-off angle. The expressions for $v(\theta), h_{\text {take-off }}(\theta)$, $h_{\text {landing }}(\theta), d_{\text {take-off }}(\theta)$, and $d_{\text {landing }}(\theta)$ were obtained by fitting mathematical relations to plots of take-off speed, take-off height, landing height, take-off distance, and landing distance as a function of take-off angle. The selected mathematical relations were based on physical models, but also had an element of empirical fitting. Only two or three fitted parameters were used in the mathematical relations so as to minimise the uncertainty associated with the fitted parameter values.

\subsection{Participants and jumping protocol}

Five physically active males volunteered to participate in the study (Table 1). The study was approved by the Human Ethics Committee of the University of Sydney, the participants were informed of the protocol and procedures prior to their involvement, and written consent to participate was obtained. All jumps were performed in a long jump pit. The take-off was performed from a flat, dry, synthetic running track (Mondo Super X Performance), and the sand landing area was level with the take-off surface. The participants wore a black bodysuit and their own training shoes. The participants were instructed to use a self-selected jumping technique, with the only restrictions being that at the start of the jump the feet must be behind the take-off line, there must be no movement of the feet prior to take-off, and the jumper must retain balance after landing without falling backwards into the pit. All five participants elected to use a pronounced countermovement and a double-arm swing. Arm swing improves performance in a standing long jump by $10-20 \%$ above that produced without arm swing (Ashby \& Heegaard, 2002; Davies \& Jones, 1993). 
Table 1

Age, height, and mass of the participants

\begin{tabular}{llll}
\hline Participant & Age (years) & Height $(\mathrm{m})$ & Mass $(\mathrm{kg})$ \\
\hline 1 & 21 & 1.73 & 75 \\
2 & 28 & 1.74 & 75 \\
3 & 23 & 1.69 & 82 \\
4 & 25 & 1.83 & 72 \\
5 & 24 & 1.87 & 86 \\
\hline
\end{tabular}

Each participant performed five maximum-effort standing long jumps at his preferred takeoff angle, and then twenty-five maximum-effort jumps at five other take-off angles that were "vertical $\left(90^{\circ}\right)$ " and "much higher", "higher", "lower", and "much lower" than his preferred take-off angle. The order of the five other take-off angles for each participant was altered to preclude any effect resulting from the order. A 2-minute rest interval was given between jumps to minimise the effects of fatigue on jump performance.

\subsection{Video analysis}

The jump distances were measured to the nearest centimetre using a fibreglass tape measure, and two JVC GR-SZ7000 compact S-VHS camcorders operating at $50 \mathrm{~Hz}$ were used to record the jumper's movements at take-off and at landing. The video cameras were mounted on rigid tripods and placed at right angles to the jump direction about $10 \mathrm{~m}$ away from the take-off line and landing pit. The optical axis was adjusted to coincide with the centre of mass of the jumper at the instant of takeoff or landing, and the field of view was zoomed to maximise the image size. The movement space of each camera was calibrated with a $1.30 \times 1.30$-m calibration object that was placed along the midline of the jumping movement.

A standing long jump is a symmetrical activity, and so a two-dimensional sixsegment model of the jumper which combined left and right limb segments was used for analysing the kinematic variables. Seven white markers $(2.5 \mathrm{~cm}$ in diameter) were fixed to the right side of the bodysuit with adhesive tape at selected bony landmarks. An Ariel Performance Analysis System was used to automatically digitise the video images. The segmental data used were those proposed by Dempster (1955) for male adults, and the two-dimensional coordinates of the body landmarks and the participant's centre of mass were calculated from the digitised data using the direct linear transform (DLT) algorithm. Coordinate data were smoothed using a second-order Butterworth digital filter with a cut-off frequency of $5 \mathrm{~Hz}$, and the velocity of the participant's centre of mass was calculated by direct differentiation of the coordinate data. The choice of cut-off frequency was based on a residual analysis (Winter, 1990) and a visual inspection of the power spectra of the coordinate and velocity data.

The instant of take-off was defined as the first clear frame in which the jumper's feet were observed to break contact with the ground (Hay, Miller, \& 
Cantera, 1986), and the instant of landing was defined as the first clear frame in which the jumper's feet were observed to contact the landing pit. The jumper's take-off speed and take-off angle were calculated from the horizontal and vertical speed of the jumper's centre of mass at the instant of take-off, and the take-off height and take-off distance were the vertical and horizontal distances of the jumper's centre of mass relative to the take-off line. Likewise, the landing height and landing distance were the vertical and horizontal distances of the jumper's centre of mass relative to the location of the heels at the instant of landing.

\section{Results}

Table 2 lists the average values of the take-off and landing variables for the jumps at the participant's preferred take-off angle. The values of jump distance, take-off speed, and take-off angle are similar to the values for physically active young men reported in other studies of the standing long jump (Aguado et al., 1997; Ashby \& Heegaard, 2002; Horita et al., 1991; Izquierdo, Aguado, Ribas, Linares, Vila, Voces, Alvarez \& Prieto, 1998). Figs. 2-4 show the take-off and landing variables as a function of the take-off angle for Participant 1. Plots similar to those in Figs. 2-4 were obtained for the other four participants.

Table 2

Take-off and landing variables for the jumps at the participant's preferred take-off angle $(n=5$, mean $\pm s)$.

\begin{tabular}{llllllll}
\hline Participant & $\begin{array}{l}\text { Jump } \\
\text { distance } \\
d_{\text {jump }}(\mathrm{m})\end{array}$ & $\begin{array}{l}\text { Take-off } \\
\text { speed } \\
v\left(\mathrm{~m} \cdot \mathrm{s}^{-1}\right)\end{array}$ & $\begin{array}{l}\text { Take-off } \\
\text { angle } \\
\theta\left({ }^{\circ}\right)\end{array}$ & $\begin{array}{l}\text { Take-off } \\
\text { height } \\
h_{\text {take-off }}(\mathrm{m})\end{array}$ & $\begin{array}{l}\text { Take-off } \\
\text { distance } \\
d_{\text {take-off }}(\mathrm{m})\end{array}$ & $\begin{array}{l}\text { Landing } \\
\text { height } \\
h_{\text {landing }}(\mathrm{m})\end{array}$ & $\begin{array}{l}\text { Landing } \\
\text { distance } \\
d_{\text {landing }}(\mathrm{m})\end{array}$ \\
\hline 1 & $2.33 \pm 0.08$ & $3.40 \pm 0.07$ & $33.3 \pm 1.1$ & $0.93 \pm 0.03$ & $0.66 \pm 0.03$ & $0.59 \pm 0.02$ & $0.18 \pm 0.04$ \\
2 & $2.13 \pm 0.03$ & $3.39 \pm 0.10$ & $39.1 \pm 2.4$ & $0.92 \pm 0.04$ & $0.58 \pm 0.02$ & $0.74 \pm 0.03$ & $0.20 \pm 0.02$ \\
3 & $2.14 \pm 0.04$ & $3.31 \pm 0.05$ & $34.3 \pm 2.3$ & $0.92 \pm 0.01$ & $0.57 \pm 0.05$ & $0.69 \pm 0.01$ & $0.21 \pm 0.02$ \\
4 & $2.34 \pm 0.08$ & $3.55 \pm 0.11$ & $31.4 \pm 2.8$ & $0.95 \pm 0.02$ & $0.72 \pm 0.05$ & $0.64 \pm 0.03$ & $0.16 \pm 0.04$ \\
5 & $2.20 \pm 0.12$ & $3.24 \pm 0.13$ & $35.1 \pm 1.9$ & $1.04 \pm 0.02$ & $0.60 \pm 0.04$ & $0.79 \pm 0.04$ & $0.26 \pm 0.02$ \\
\hline
\end{tabular}




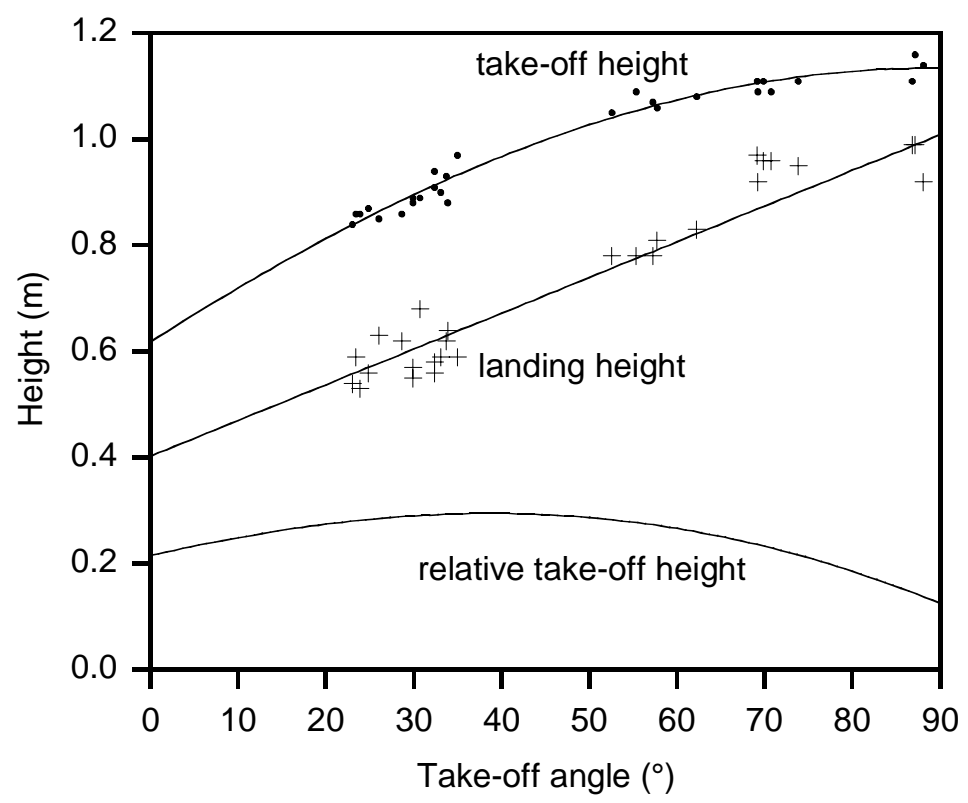

Fig. 2. Take-off height and landing height for Participant 1. The fitted curves are from Eqs. (6) and (8). The relative take-off height is the difference between the take-off height and the landing height.

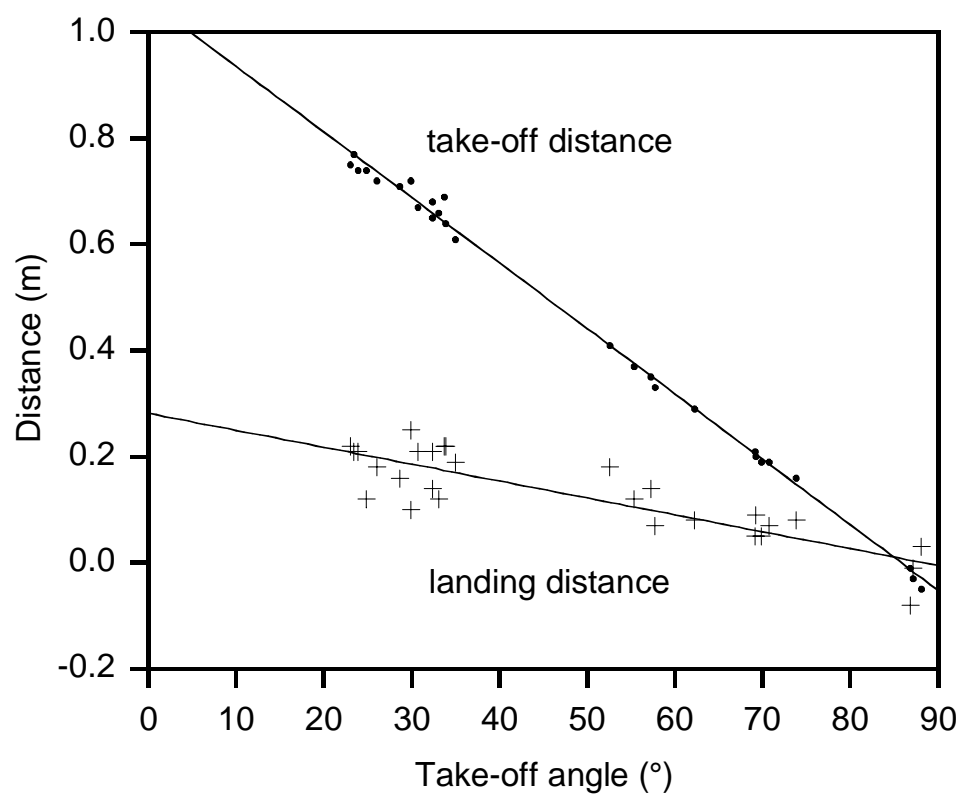

Fig. 3. Take-off distance and landing distance for Participant 1. The fitted curves are from Eqs. (7) and (9). 


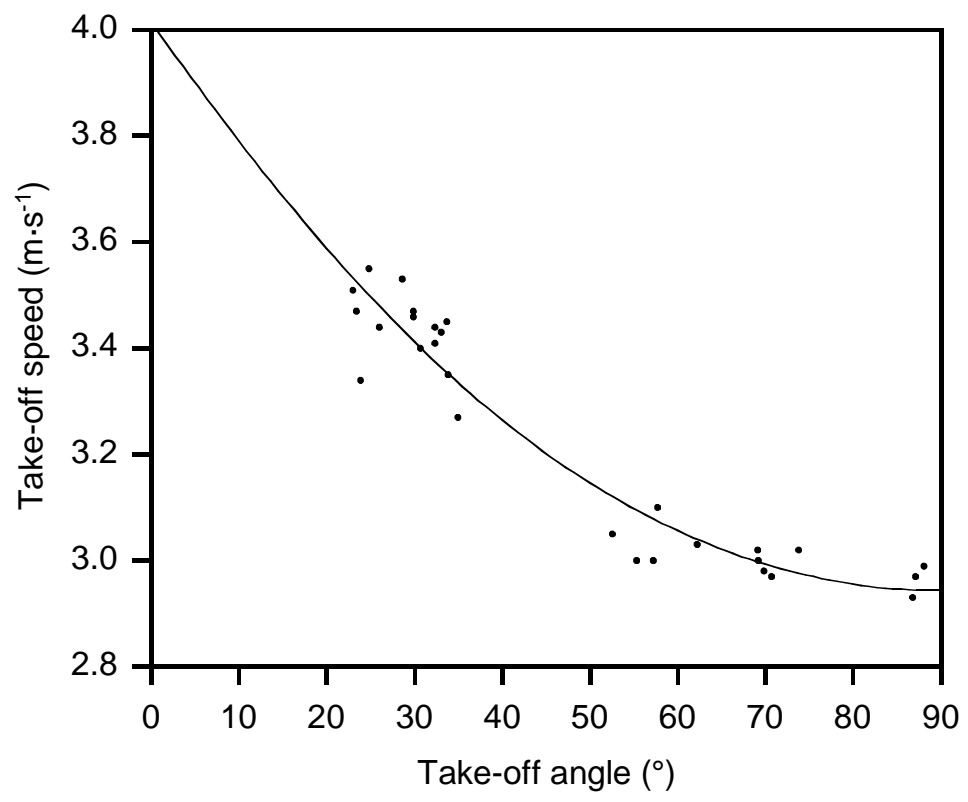

Fig. 4. At higher take-off angles the jumper spent a greater fraction of his muscular force overcoming body weight and so the take-off speed decreased with increasing take-off angle. Data for Participant 1 and fitted curve from Eq. (10).

\subsection{Take-off height and take-off distance}

The take-off height and take-off distance were determined by the jumper's body configuration, angle of forward lean at take-off, and take-off angle (Fig. 5). At the instant of take-off the jumper had approximately the same body configuration at all take-off angles, and so the expressions for the take-off height and take-off distance were of the form

$$
\begin{aligned}
& h_{\text {take-off }}=L \sin \alpha \\
& d_{\text {take-off }}=L \cos \alpha,
\end{aligned}
$$

where $L$ is the distance from take-off line to the jumper's centre of mass at the instant of take-off, and $\alpha$ is the angle of forward lean (Fig. 5). The angle of forward lean is not the same as the take-off angle, but for all jumpers there was a strong linear relation $\left(r^{2}=0.99\right)$ between the angle of forward lean and the take-off angle. The angle of forward lean was about $45^{\circ}$ at the lowest take-off angles (about $20^{\circ}$ ), and increased steadily to about $90^{\circ}$ at a take-off angle of $90^{\circ}$. The take-off height and take-off distance may then be expressed by

$$
\begin{aligned}
& h_{\text {take-off }}(\theta)=L \sin \left[\alpha_{\mathrm{p}}+A\left(\theta-\theta_{\mathrm{p}}\right)\right], \\
& d_{\text {take-off }}(\theta)=L \cos \left[\alpha_{\mathrm{p}}+A\left(\theta-\theta_{\mathrm{p}}\right)\right],
\end{aligned}
$$

where $\alpha_{\mathrm{p}}$ is the angle of forward lean at the jumper's preferred take-off angle, $\theta_{\mathrm{p}}$, and $A$ is the rate of increase in forward lean with increasing take-off angle. 


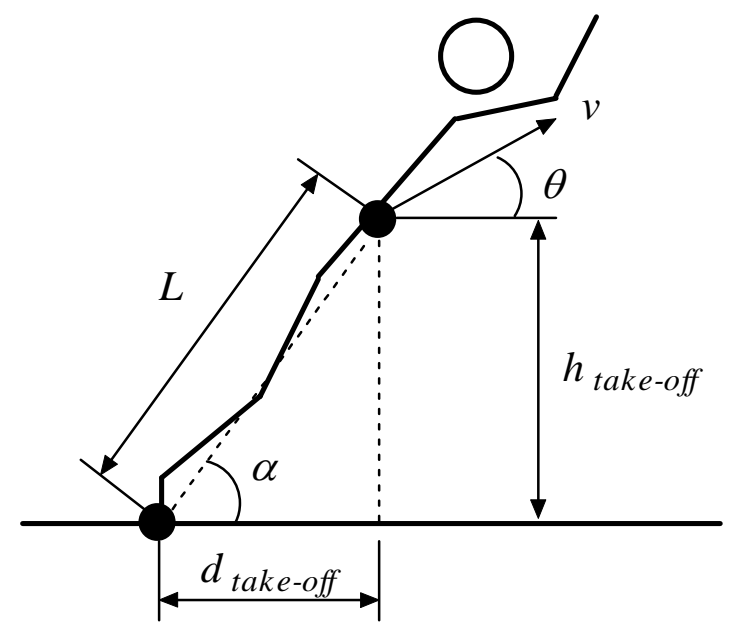

Fig. 5. Simple geometrical model of the jumper at take-off showing the relation between the angle of forward lean and the take-off height and take-off distance.

Curves of the form of Eqs. (6) and (7) were fitted to plots of the jumper's take-off height and take-off distance as a function of take-off angle by selecting values of $L, \alpha_{\mathrm{p}}$, and $A$ using the Levenberg-Marquardt algorithm (Press, Flannery, Teukolsky, \& Vetterling, 1988). For Participant 1 the calculated values and standard errors were $L=1.13 \pm 0.01 \mathrm{~m}, \alpha_{\mathrm{p}}=54 \pm 1^{\circ}$, and $A=0.64 \pm 0.04$. Figs. 2 and 3 show the fitted curves for Participant 1 . Similar values and curves were obtained for the other four participants.

The distance from the take-off line to the jumper's centre of mass at take-off, $L$, was about $62-65 \%$ of the jumper's standing height. All five jumpers adopted a similar body configuration at take-off, and this configuration was similar to that used when performing a standing vertical jump with arm swing. The values of $L$ were therefore similar to reported values for the height of the centre of mass at takeoff in the standing vertical jump (Feltner, Fraschetti, \& Crisp, 1999; Harman, Rosenstein, Frykman, \& Rosenstein, 1990).

\subsection{Landing height and landing distance}

The landing height, $h_{\text {landing, }}$ and landing distance, $d_{\text {landing, }}$, depended on the take-off angle and the jumper's body configuration at landing. The body configuration adopted by a jumper at landing was determined by the requirement to maintain balance after landing. When jumping at low take-off angles the jumper had a high horizontal speed and so he could land with his feet far ahead of his body without the risk of falling backward after landing (Fig. 1). When landing from a high take-off angle the jumper had a lower horizontal speed and so landed in a nearly upright position with the feet only slightly ahead of the body. Therefore, the landing height steadily increased and the landing distance steadily decreased as the take-off angle increased. The plots of landing height and landing distance versus take-off angle (Figs. 2 and 3) suggested linear relations of the form

$$
\begin{aligned}
& h_{\text {landing }}(\theta)=h_{\mathrm{p}}+B\left(\theta-\theta_{\mathrm{p}}\right), \\
& d_{\text {landing }}(\theta)=d_{\mathrm{p}}-C\left(\theta-\theta_{\mathrm{p}}\right),
\end{aligned}
$$


where $h_{\mathrm{p}}$ is the landing height at the jumper's preferred take-off angle, $B$ is a constant that determines the rate of increase in landing height with increasing takeoff angle, $d_{\mathrm{p}}$ is the landing distance at the jumper's preferred take-off angle, and $C$ is a constant that determines the rate of decrease in landing distance with increasing take-off angle.

Curves of the form of Eqs. (8) and (9) were fitted to plots of the jumper's landing height and landing distance as a function of take-off angle by selecting values of $h_{\mathrm{p}}, d_{\mathrm{p}}, B$, and $C$ using the Levenberg-Marquardt algorithm (Press et al., 1988). For Participant 1 the calculated values and standard errors were $h_{\mathrm{p}}=0.63 \pm$ $0.01 \mathrm{~m}, d_{\mathrm{p}}=0.18 \pm 0.01 \mathrm{~m}, B=0.0067 \pm 0.0004 \mathrm{~m} \cdot$ degree $^{-1}$, and $B=0.0032 \pm$ $0.0003 \mathrm{~m} \cdot$ degree $^{-1}$. Figs. 2 and 3 show the fitted curves for Participant 1. Similar values and curves were obtained for the other four participants.

\subsection{Relative take-off height}

In the standing long jump, the flight distance is determined by the relative take-off height, $h(\theta)$, which is the height difference between the take-off and the landing. For the five participants in this study, the relative take-off height showed little variation with changes in take-off angle (Fig. 2).

\subsection{Take-off speed}

The take-off action in the standing long jump is a moderately complex movement. The jumper flexes at the hips and knees in a downward countermovement, rotates his body about his feet to the desired amount of forward lean, and then performs an explosive leg extension to project his body outwards and upwards (Ridderikhoff, Batelaan, \& Bobbert, 1999). The jumper also uses a coordinated forward and upward swing of the arms to increase his speed during the leg extension phase of the jump (Ashby \& Heegaard, 2002). A simple model of the take-off was devised in an attempt to explain the observed decrease in take-off speed with increasing take-off angle (Fig. 4). The take-off action was reduced to just the leg extension (or "push-off") phase. The jumper was assumed to apply a constant force, $F$, to the centre of mass at a constant angle to the horizontal. The combined effect of the applied force and the jumper's body weight is a resultant force that produces acceleration of the centre of mass along a straight line path, $l$, at an angle $\theta$ to the horizontal. This acceleration path is at the same angle to the horizontal as the take-off angle.

During the push-off phase the force generated by the jumper must overcome both his body weight and his inertia. At higher take-off angles the jumper spends a greater fraction of his muscular force overcoming body weight, and so less force is available to accelerate his body. That is, more of the work performed by the jumper's muscles is required to increase the gravitational potential energy of the centre of mass, at the expense of increasing the kinetic energy of the centre of mass. The take-off speed at high take-off angles is therefore not as great as at low take-off angles.

Applying the law of conservation of energy to the push-off phase gives an expression for the take-off speed as a function of take-off angle (Linthorne, 2001): 


$$
v(\theta)=\left\{\frac{2 F l}{m}\left[1-\left(\frac{m g}{F}\right)^{2} \sin ^{2}\left(90^{\circ}+\theta\right)\right]^{1 / 2}-2 g l \sin \theta\right\}^{1 / 2},
$$

where $m$ is the mass of the jumper. In this model we have assumed the force exerted by the jumper is the same for all take-off angles, and the speed of the jumper's centre of mass at the start of the push-off phase is approximately zero.

A curve of the form of Eq. (10) was fitted to a plot of the jumper's take-off speed as a function of take-off angle by selecting values of $F$ and $l$ using the Levenberg-Marquardt algorithm (Press et al., 1988). For Participant 1 the calculated values and standard errors were $F=1330 \pm 60 \mathrm{~N}$ and $l=0.54 \pm 0.04 \mathrm{~m}$. Fig. 4 shows the fitted curve for Participant 1 . Similar values and curves were obtained for the other four participants. The calculated values of $l$ are consistent with the average value of the push-off range obtained from the video data (e.g. 0.58 $\pm 0.02 \mathrm{~m}$ for Participant 1 ), and the calculated values of $F$ are consistent with force platform measurements of the peak ground reaction force in the standing long jump (Aguado et al., 1997; Horita et al., 1991).

Although Eq. (10) gives a good fit to the experimental data and the fitted values of $F$ and $l$ appear to have some correspondence to physical reality, the model behind the equation is simplistic. The take-off force generated by the jumper is not constant, as assumed in the model. The force generated by the jumper changes throughout the take-off because of the changing lengths, moment arms, and contraction speeds of the jumper's muscles (Ashby \& Heergaard, 2002; Ridderikhoff et al., 1999). Also, we have no experimental evidence that the force exerted by the jumper is the same for all take-off angles, and our experiments showed that the speed of the jumper's centre of mass at the start of the push-off phase steadily increases at lower take-off angles. Therefore, the practical value of the simple model used here should not be extended beyond the ability to provide a good empirical fit to the take-off speed versus take-off angle data.

\subsection{The optimum take-off angle}

The optimum take-off angle for each jumper was calculated and then compared to his preferred take-off angle. The values of $L, \alpha$, and $A$ for the jumper were substituted into Eq. (6), the values of $h_{\mathrm{p}}$ and $B$ were substituted into Eq. (8), and the values of $F$ and $l$ were substituted into Eq. (10). The resulting expressions for $v(\theta)$ and $h(\theta)$ were then substituted into Eq. (2) to give an expression for the flight distance, $d_{\text {flight }}(\theta)$. The values of $L, \alpha_{\mathrm{p}}, A, d_{\mathrm{p}}$, and $C$ were substituted into Eqs. (7) and (9) to give expressions for the take-off distance, $d_{\text {take-off }}(\theta)$, and the landing distance, $d_{\text {landing }}(\theta)$. The three component distances were added to give the jump distance (Eq. 1), and the jump distance was plotted as a function of the takeoff angle. The optimum take-off angle was the point on the curve at which the jump distance was greatest (Fig. 6). 


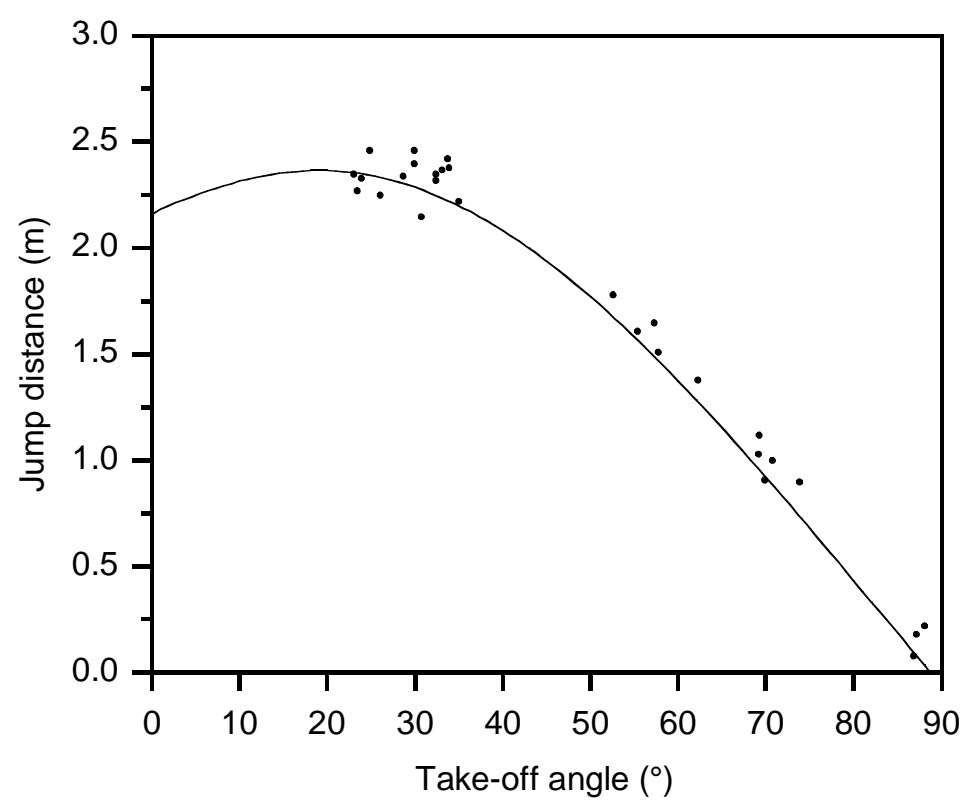

Fig. 6. Calculated jump distance as a function of take-off angle. The optimum take-off angle is the angle at which the jump distance is greatest. Also shown are the measured jump distances. The calculated optimum take-off angle is about $19^{\circ}$, but the jumper's preferred take-off angle was about $33^{\circ}$

Fig. 7 illustrates how the relations for $v(\theta), h(\theta), d_{\text {take-off }}(\theta)$, and $d_{\text {landing }}(\theta)$ contribute to the optimum take-off angle. The optimum take-off angle will be $45^{\circ}$ if a jumper can produce the same take-off speed at all take-off angles and if the landing height is the same as the take-off height. However, as the take-off angle increases the take-off force exerted by the jumper must overcome a greater fraction of body weight and so the take-off speed decreases (Fig. 4). For the participants in this study, the decrease in take-off speed with increasing take-off angle lowered the optimum take-off angle by $4-7^{\circ}$. This is illustrated by the dashed line in Fig. 7 , where the flight distance of the jumper has been calculated by substituting the jumper's expression for $v(\theta)$ into the equation for a projectile in free flight (Eq. 2), while assuming a zero height difference between the take-off and landing (i.e. $h=$ 0 ). Although the take-off height and landing height both increased with increasing take-off angle, the height difference between take-off and landing remained approximately constant at about 10-30 cm (Fig. 2). This height difference lowered the optimum take-off angle by a further $4-8^{\circ}$. The combined effects of $v(\theta)$ and $h(\theta)$ are illustrated by the curve labelled 'flight distance' in Fig. 7. Both the takeoff distance and landing distance decreased with increasing take-off angle, and these relations reduced the optimum take-off angle by about $8^{\circ}$ and $2^{\circ}$ respectively. The effects of the take-off distance and landing distance on the optimum take-off angle are illustrated in Fig. 7 by the difference between the curves labelled 'flight distance' and 'jump distance'. 


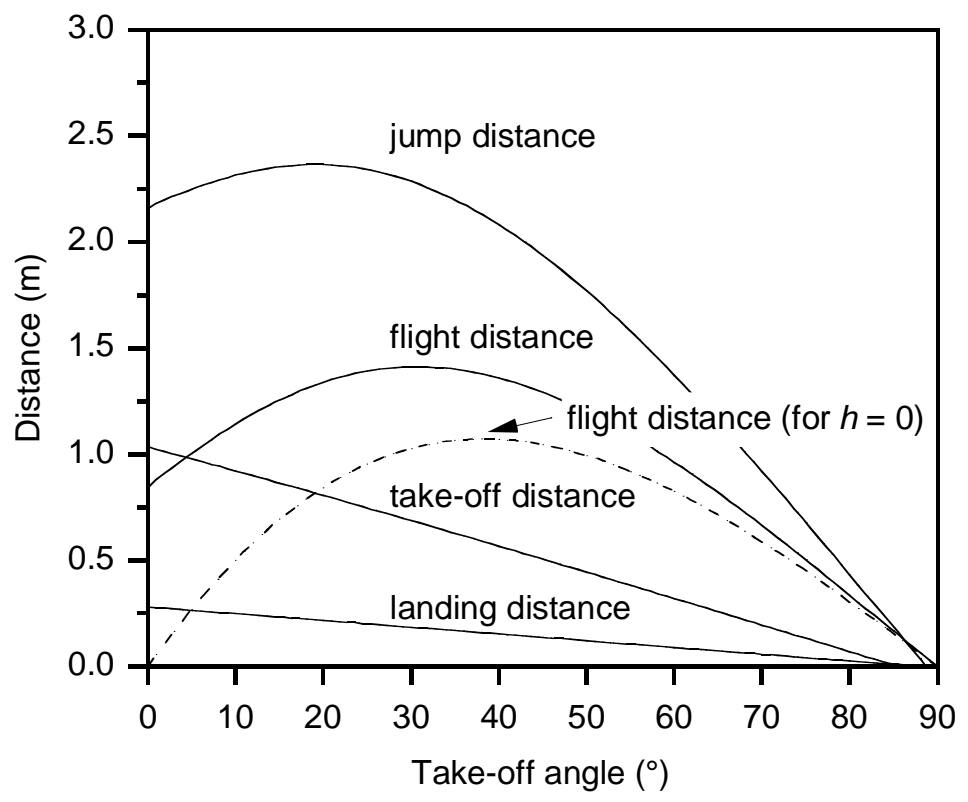

Fig. 7. The jump distance is the sum of the take-off distance, flight distance, and landing distance. The dashed line shows the calculated flight distance for a relative take-off height of $h=0$. Calculations for Participant 1

Table 3 lists the calculated optimum take-off angles for the five jumpers. The standard error associated with the calculated optimum take-off angle was determined using the quadrature method of combining errors (Taylor, 1997). The method of calculating the optimum take-off angle used here was expected to produce values that were in good agreement with the jumper's preferred take-off angle. However, for all five jumpers the calculated optimum take-off angles were lower than the jumper's preferred take-off angle.

\section{Table 3}

Comparison of the calculated optimum take-off angle with the participant's preferred takeoff angle (value \pm standard error)

\begin{tabular}{ccc}
\hline Participant & $\begin{array}{c}\text { Calculated optimum } \\
\text { take-off angle }\left(^{\circ}\right)\end{array}$ & $\begin{array}{c}\text { Preferred } \\
\text { take-off angle }\left(^{\circ}\right)\end{array}$ \\
\hline 1 & $19.1 \pm 5.8$ & $33.3 \pm 0.5$ \\
2 & $25.8 \pm 5.8$ & $39.1 \pm 1.1$ \\
3 & $22.2 \pm 4.5$ & $34.3 \pm 1.0$ \\
4 & $24.3 \pm 3.5$ & $31.4 \pm 1.3$ \\
5 & $26.7 \pm 5.4$ & $35.1 \pm 1.0$ \\
\hline
\end{tabular}




\section{Discussion}

Although the participants were instructed to perform jumps using take-off angles that were "lower" and "much lower" than their preferred take-off angle, the minimum take-off angles used were only $18-29^{\circ}$. We suggest that the participants could have jumped at lower take-off angles if they had worn spiked shoes to increase traction at take-off. To perform a successful jump using a very low takeoff angle the jumper would have to split his legs out wide to either side of his body so as to give his feet sufficient ground clearance during the flight phase of the jump.

Using spiked shoes to increase traction at take-off would have provided additional jump data at very low take-off angles, and resulted in a more precise calculation of the optimum take-off angle. In this study the calculated optimum take-off angle was strongly dependent on the shape of the curve that was fitted to the take-off speed data, particularly in the region from 0 to $25^{\circ}$ where there was no little or no experimental data (Fig. 4). A fitted curve that exhibited a less rapid rate of increase in this region would have produced a higher calculated optimum take-off angle, and hence closer agreement with the jumper's preferred take-off angle.

Although the jumpers in this study did not jump at their calculated optimum take-off angle, the distance lost due to a sub-optimum take-off angle was relatively small; only about 4-14 cm. Projecting the body at the optimum take-off angle is not very important in a successful standing long jump. The jump distance is not sensitive to take-off angle and so relatively large errors in take-off angle can be tolerated. For the jump distance to be within $2 \mathrm{~cm}$ of the maximum achievable distance, the take-off angle must be within about $5^{\circ}$ of the optimum take-off angle (Fig. 6). This accuracy is in contrast to the running long jump, where the curve of jump distance versus take-off angle is more sharply peaked and a tolerance of only about $1^{\circ}$ is allowable for a similar relative loss in performance (Linthorne et al., 2005).

It is much more important for a jumper to attain a high take-off speed than to jump at the optimum take-off angle. In this study, distance lost owing to variations take-off angle was less than distance lost owing to variations in take-off speed. When Participant 1 was jumping at his preferred take-off angle, inter-trial variations in take-off angle produced changes in jump distance of about $5 \mathrm{~cm}$, whereas intertrial variations in take-off speed produced changes in jump distance of about $25 \mathrm{~cm}$.

\section{Conclusions}

The optimum take-off angle in the standing long jump is considerably less than $45^{\circ}$. The three main influences that reduce the optimum take-off angle to below $45^{\circ}$ are the relation between the take-off speed and take-off angle, the 10-30 $\mathrm{cm}$ height difference between take-off and landing, and the relation between takeoff distance and take-off angle. In the standing long jump the take-off speed decreases with increasing take-off angle because the jumper must expend a greater fraction of the take-off force in overcoming their body weight. For the five participants in this study the calculated optimum take-off angles were $19-27^{\circ}$.

An unexpected finding in this study was that the participants preferred to use a take-off angle that was higher than the calculated optimum take-off angle. However, the loss in jump distance through using a sub-optimum take-off angle was relatively small. We suggest that the participants could have jumped with a lower 
take-off angle and achieved a slightly greater jump distance if they had worn spiked shoes to increase traction at take-off, and if they had jumped using a sideways split of the legs to allow a greater clearance of the feet during the flight phase.

\section{References}

Aguado, X., Izquierdo, M., \& Montesinos, J. L. (1997). Kinematic and kinetic factors related to the standing long jump performance. Journal of Human Movement Studies, 32, 157-169.

Ashby, B. M., \& Heegaard, J. H. (2002). Role of arm motion in the standing long jump. Journal of Biomechanics, 35, 1631-1637.

Davies, B. N., \& Jones, K. G. (1993). An analysis of the performance of male students in the vertical and standing long jump test and the contribution of arm swing. Journal of Human Movement Studies, 24, 25-38.

Dempster, W. T. (1955). Space requirements of the seated operator. (WADC-TR55-159) Wright-Patterson Air Force Base, OH.

Feltner, M. E., Fraschetti, D. J., \& Crisp, R. J. (1999). Upper extremity augmentation of lower extremity kinetics during countermovement vertical jumps. Journal of Sports Sciences, 17, 449-466.

Harman, E. A., Rosenstein, M. T., Frykman, P. N., \& Rosenstein, R. M. (1990). The effects of arms and countermovement on vertical jumping. Medicine and Science in Sports and Exercise, 22, 825-833.

Hay, J. G., Miller, J. A., \& Cantera, R. W. (1986). The techniques of elite male long jumpers. Journal of Biomechanics, 19, 855-866.

Horita, T., Kitamura, K., \& Kohno, N. (1991). Body configuration and joint moment analysis during standing long jump in 6-yr-old children and adult males. Medicine and Science in Sports and Exercise, 23, 1068-1077.

Hubbard, M. (2001). The flight of sports projectiles. In V. M. Zatsiorsky (Ed.), Biomechanics in sport (pp. 381-400). Oxford: Blackwell Science.

Izquierdo, M., Aguado, X., Ribas, T., Linares, F., Vila, L., Voces, J. A., Alvarez, A. I., \& Prieto, J. G. (1998). Jumping performance, isometric force and muscle characteristics in non athletic young men. Journal of Human Movement Studies, 35, 101-117.

Linthorne, N. P. (2001). Optimum release angle in the shot put. Journal of Sports Sciences, 19, 359-372.

Linthorne, N. P., Guzman, M. S., \& Bridgett, L. A. (2005). Optimum take-off angle in the long jump. Journal of Sports Sciences, 23, 703-712

Press, W. H., Flannery, B. P., Teukolsky, S. A., \& Vetterling, W. T. (1988). Numerical recipes in $C$ : The art of scientific computing. Cambridge, NY: Cambridge University Press.

Ridderikhoff, A., Batelaan, J. H., \& Bobbert, M. F. (1999). Jumping for distance: control of the external force in squat jumps. Medicine and Science in Sports and Exercise, 31, 1196-1204.

Red, W. E., \& Zogaib, A. J. (1977). Javelin dynamics including body interaction. Journal of Applied Mechanics, 44, 496-498. 
Taylor, J. R. (1997). An introduction to error analysis: the study of uncertainties in physical measurement (second ed.). Sausalito, CA: University Science Books.

Wiklander, J. \& Lysholm, J. (1987). Simple tests for surveying muscle strength and muscle-stiffness in sportsmen. International Journal of Sports Medicine, 8, 50-54.

Winter, D. A. (1990). Biomechanics and motor control of human movement (second ed.). New York: John Wiley. 